\title{
Síndrome De Cotard Y Catatonía: Reporte De Un
}

Caso.

\section{Cotard's Syndrome And Catatonia: A Case Report.}

\author{
Sergio Vergara R. ${ }^{1,2}$ Pamela Díaz ${ }^{3}$
}

\begin{abstract}
Cotard's Syndrome is a rare neuropsychiatric condition, initially described by Jules Cotard as a hypochondriacal delusion and then as Delusion of negation, in which the patient denies the existence of parts of his body, his own existence and / or the entire world. The appearance of a Catatonic Syndrome together with Cotard Syndrome is even more infrequent. We present the case of a 72-year-old patient with a psychotic depression, who developed Cotard's Syndrome and later Catatonia. She achieves good response after the addition of Lorazepam and Venlafaxine to the current pharmacological treatment, so the use of Electroconvulsive Therapy is dismissed. Total remission of symptoms and subsequent functional recovery ad integrum was observed, being evaluated through clinical interview, Hamilton Depression Rating Scale, Bush-Francis Catatonia Rating Scale and Barthel Index. In addition, other case reports on this comorbidity are reviewed, and unlike most of these, the favorable evolution of the patient stands out without the need for Electroconvulsive Therapy. The relationship between the two syndromes has not been elucidated, although some authors have proposed the hypothesis of shared neurobiological pathways and others have postulated the appearance of catatonic symptoms such as the progression of Cotard's Syndrome. To clarify these questions, more studies are needed in order to know the etiopathogenesis of this unusual combination.
\end{abstract}

Key words: Cotard Syndrome, Catatonia, Depression.

Rev Chil Neuro-Psiquiat 2020; 58 (1): 66-73

\section{Introducción}

E Síndrome de Cotard fue presentado en 1880 por Jules Cotard como un Delirio hipocondríaco en pacientes con melancolía ansiosa, para luego referirse al mismo cuadro clínico como Delirio de negación (1). Este delirio, puede expresarse desde la

Aceptado: 19/03/2020

Recibido: $17 / 12 / 2018$

Los Autores declaran no tener ningún conflicto de intereses.

Médico Psiquiatra. Universidad Católica del Maule, Facultad de Medicina, Talca, Chile.

Hospital Regional de Talca, Servicio de Psiquiatría, Talca, Chile.

Médico Residente Psiquiatría Adultos, Universidad Católica del Maule, Talca, Chile. 
negación de la existencia de diversas partes del cuerpo del paciente hasta la negación de su propia existencia, incluso del mundo entero $^{(2)}$. Además, puede culminar en una forma de delirio pseudomegalomaníaco o "Delirio de enormidad", caracterizado por ideas de inmortalidad e inmensidad. Se trata de un síndrome poco frecuente, grave, que representa alto riesgo de autoagresión y suicidioporpartedelpaciente ${ }^{(3,4)}$. Supresencia se asocia a diversos cuadros, principalmente neuropsiquiátricos, como Trastorno Bipolar, Trastorno Depresivo, Esquizofrenia, Enfermedad de Parkinson, Enfermedad cerebrovascular isquémica, Epilepsia, Hemorragia subdural, Atrofia de la corteza insular y Catatonía, entre otras ${ }^{(5)}$. Dentro de las opciones terapéuticas reportadas se incluyen Fluoxetina, Paroxetina, Carbonato de Litio, y tratamientos combinados como Haloperidol y Clomipramina. En el caso de Depresión psicótica o melancolía se ha sugerido en varios estudios el uso de Terapia Electroconvulsiva (TEC), especialmente en combinación a tratamiento psicofarmacológico de mantención post$\mathrm{TEC}^{(3)}$.

Por otra parte, la Catatonía es un cuadro neuropsiquiátrico descrito inicialmente por Karl Kahlbaum en 1874, que consiste en un síndrome psicomotor en el que se identifican un subtipo "inhibido" y otro "excitado". El primero es más frecuente y se caracteriza por inmovilidad, mutismo, mirada fija, rigidez, entre otros. El segundo subtipo es menos prevalente y se desarrollan períodos de agitación psicomotora. Es reconocida la aparición del síndrome en un amplio espectro de enfermedades médicas y trastornos psiquiátricos, especialmente en los trastornos del ánimo ${ }^{(6)}$. Su patogenia aún no se ha dilucidado, aunque se reconoce la influencia de los sistemas de neurotransmisión gabaérgico y glutamatérgico. En cuanto al pronóstico y tratamiento, es una condición que pone en riesgo la vida de quienes lo padecen, y que se beneficia de dosis bajas de benzodiazepinas y Terapia Electroconvulsiva (TEC) $)^{(7)}$.

La aparición de síntomas catatónicos en el Sindrome de Cotard es poco frecuente, y solo se han encontrado algunos reportes de caso que ilustran dicha coexistencia ${ }^{(2,5,8)}$.

Con el fin de aportar al conocimiento de esta comorbilidad y su manejo, se presenta a continuación el caso de una mujer de 72 años, que en el curso de un Episodio Depresivo mayor grave con síntomas psicóticos, desarrolla un Sindrome de Cotard y posteriormente una Catatonía.

\section{Caso Clínico}

Paciente de 72 años, sexo femenino, educación técnico profesional, jubilada, casada y madre de dos hijos. En control médico por Hipertensión Arterial, sin antecedentes psiquiátricos previos ni consumo problemático de sustancias, con capacidad conservada para realizar actividades de la vida diaria premórbido y sin enfermedades psiquiátricas en la familia.

Dos meses antes de la hospitalización, debuta con insomnio de despertar precoz, apetito disminuido, menor frecuencia de las deposiciones y angustia acentuada.

Luego, se suman ideas delirantes nihilistas de no existencia, ausencia de órganos e ideas de ruina; señala que murió, no existe en este mundo y necesita que su médico le extienda un certificado de defunción. Reporta que no posee estómago y por lo tanto no tiene sentido comer si ella no puede tragar, agrega que no siente palpitar su corazón porque no lo tiene. Además, presenta alucinaciones visuales: "veo bebés colgando como granos de uva, y el cielo completamente rojo". Se suma ideación suicida de corta duración en una oportunidad, con sensación de control parcial.

En las semanas previas a la hospitalización, cursa con progresivo retardo psicomotor, disminución del impulso y dependencia 
para desarrollar las actividades de la vida cotidiana.

Ambulatoriamente inicia Fluoxetina y Olanzapina alcanzando dosis plena, sin respuesta, e ingresa a la Unidad de Hospitalización de Cuidados Intensivos en Psiquiatría (UHCIP) para estudio y manejo de Episodio Depresivo Mayor grave con síntomas psicóticos y Síndrome de Cotard. Se constata rigidez y temblor leve de extremidades superiores, dificultad en la marcha, bloqueo del pensamiento, orientada temporo-espacialmente, memoria reciente conservada, pensamiento abstracto, y persistencia de los delirios de negación y sintomatología melancólica. Al ingreso obtiene 39 puntos en la Hamilton Depression Rating Scale (HDRS) compatible con Depresión muy severa; y 5 puntos al ser aplicado el Índice de Barthel concordante con una dependencia severa desde el punto de vista funcional. Durante los primeros días de hospitalización, se realiza estudio del caso con solicitud de exámenes para descartar otros diagnósticos, se mantiene tratamiento psicofarmacológico junto a psicoterapia de apoyo. Sus exámenes generales se encuentran dentro de rangos normales, VIH negativo, Resonancia Nuclear Magnética de cerebro que informa cambios involutivos corticales y subcorticales microangiopáticos, con Electroencefalograma normal, Punción lumbar normal, Electrocardiograma y Ecocardiograma bidimensional que muestran hipertrofia ventricular izquierda.

La evaluación por Neurología plantea como hipótesis una Demencia de etiología aún no precisada, estudio no concluyente ante dudas sobre la correlación entre la clínica de la paciente y los hallazgos en las imágenes cerebrales. Evoluciona con negativismo activo, estereotipias verbales y motoras, escasa fluencia verbal, hipocinesia y estupor. Se plantea la presencia de un Síndrome Catatónico asociado a la Psicosis Afectiva descrita, con puntuación en la Bush-Francis
Catatonia Rating Scale (BFCRS) de 7/14 en screening y de 15/69 en severidad.

Se adiciona Lorazepam $2 \mathrm{mg}$ para manejo de la sintomatología catatónica, inicia Venlafaxina para establecer tratamiento de combinación del cuadro depresivo grave y se realiza derivación a centro de referencia para Terapia Electroconvulsiva (TEC). La paciente responde al tratamiento de la sintomatología catatónica a las 24 horas de iniciado Lorazepam. La reducción de la sintomatología depresiva y de las ideas delirantes del Síndrome de Cotard se inician a los 10 días de agregados Lorazepam (3 mgs/día) y Venlafaxina (150 mgs/día), alcanzando buena respuesta a las 4 semanas. Tras dos semanas de lograda dicha respuesta, se ajusta Venlafaxina a $225 \mathrm{mgs}$ día y se hace efectivo el traslado a centro de referencia para la realización de TEC.

En dicho centro es hospitalizada por 20 días, registrándose en epicrisis la favorable evolución de la paciente con la farmacoterapia, desestimando la necesidad de TEC, mantiendo el tratamiento combinado antidepresivo descrito junto a Lorazepam y Quetiapina en dosis bajas, con indicación de seguimiento ambulatorio.

Al ser controlada en el policlínico de especialidad, se observa la remisión de la sintomatología depresiva, por otra parte, los familiares reportan que ha recuperado su nivel de funcionmiento premórbido. A los 6 meses de seguimiento, puntúa 0 tanto en el screening como en la escala de severidad de la BFCRS, obtiene 1 punto en la HDRS (no deprimida) y 100 puntos (puntuación máxima sobre la capacidad de realizar actividades de la vida diaria) en el Índice de Barthel.

\section{Discusión}

Se ha presentado el caso de una paciente adulta mayor, que en el contexto de una Depresión melancólica desarrolla intensas ideas delirantes nihilistas y alucinaciones visuales aisladas. Es hospitalizada al 
no conseguir respuesta a tratamiento antidepresivo y antipsicótico en contexto ambulatorio, evolucionando con síntomas catatónicos que responden rápidamente a la adición de una benzodiazepina (Lorazepam). En cuanto a la sintomatología depresiva psicótica del Síndrome de Cotard, se aprecia disminución de ésta a los diez días de la adición de Venlafaxina y Lorazepam, logrando buena respuesta al mes, por lo que se desestimó la aplicación de TEC en el centro de referencia. Tras la hospitalización, presenta remisión total de sintomas, con recuperación funcional total constatada clínicamente, a través de instrumentos y el reporte de familiares cercanos. Aunque el cuadro clínico y los hallazgos neuroimagenológicos inicialmente orientaban a la presencia de algún tipo de Demencia, esta hipótesis se fue descartando tras las evaluaciones por Neurología considerando la favorable evolución de la paciente. Aún así, se estima necesario mantener una perspectiva longitudinal del caso, con seguimiento estricto y valoraciones seriadas desde el punto de vista cognitivo.

La riqueza psicopatológica con que Cotard describió el Síndrome dista mucho del uso dado en las últimas décadas, en que ha sido reducido a un delirio nihilista.

Una excepción a lo señalado, es el estudio de Berrios y Luque (1995), en el que caracterizaron el Síndrome de Cotard tomando 100 casos de la literatura existente. Encontraron Depresión en el $89 \%$ de la muestra, los delirios nihilistas más frecuentes fueron los que se relacionaban con el cuerpo (86\%) y la existencia $(69 \%)$, con alta frecuencia de ansiedad, sentimientos de culpa, delirios hipocondríacos y de inmortalidad. Al analizar estadísticamente la muestra obtuvieron 3 factores: Depresión psicótica, Cotard tipo I y Cotard tipo II. El primer grupo se caracterizó por el predominio de melancolía con unos pocos delirios nihilistas. Los pacientes Cotard tipo I presentaron delirios hipocon- dríacos, nihilistas del cuerpo, concepto y existencia, que los autores señalaron como el tipo "puro" del Síndrome y su origen estaría en las delusiones y no en los trastornos afectivos, con las implicancias terapéuticas que esto podría acarrear. Por último, el grupo designado Cotard tipo II congregó ansiedad, depresión y alucinaciones auditivas constituyendo un tipo "mixto"(9).

Longitudinalmente, Yamada, Katsuragi y Fujii (1999) describieron tres etapas para el Síndrome de Cotard: "Germinación" con síntomas depresivos e hipocondría como parte de las manifestaciones prodrómicas, "Afloramiento" en que se desarrolla plenamente el Síndrome con la presencia de los delirios que lo caracterizan, y "Crónica" con dos variantes: un tipo depresivo que presenta síntomas afectivos persistentes y un tipo paranoide con sistematización de los delirios (10).

Estas observaciones proveen información valiosa y sugieren modos de categorizar casos que cursen un Síndrome de Cotard, pero no logramos contar aún con criterios definitivos e instrumentos para evaluar el síndrome. Esto limita nuesto conocimiento del cuadro, así como explica la escacez de datos epidemiológicos, la disparidad de los hallazgos neurobiológicos y las dificultades en términos de establecer diagnósticos diferenciales y esquemas de tratamiento eficaces ${ }^{(3)}$.

En el caso clínico revisado, la paciente presentó sintomatología compatible con el primer grupo descrito por Berrios y Luque (1995), es decir, una Depresión psicótica caracterizada por síntomas melancólicos con delirios nihilistas. Transitó solo por las etapas de Germinación y Afloramiento de la clasificación de Yamada et al. (1999), logrando la remisión del cuadro con el uso de tratamiento antidepresivo combinado junto a una benzodiapina y antipsicótico atípico, sin requerimiento de TEC.

Existe cierta superposición de manifestaciones clínicas entre el Síndrome 
de Cotard y la Catatonía, por lo que la evidencia de esta asociación podría ser más robusta de la que actualmente se encuentra en la literatura. Por otra parte, a menudo la Catatonía es infradiagnosticada ya que los clínicos no la pesquizan rutinariamente ${ }^{(8)}$. Sobre esta combinación, principalmente se encontraron reportes de caso, de ellos, la mayoría corresponde a Psicosis afectiva en adultos.

Dentro de los casos descritos con Trastorno Depresivo mayor con síntomas psicóticos, hallamos el reporte de un paciente de sexo masculino de 55 años que respondió a TEC, Olanzapina y Citalopram ${ }^{(11)}$. Cohen, Cottias y Basquin (1997) presentaron el caso de una mujer de 15 años que es hospitalizada por ideas delirantes nihilistas de su propia existencia y estupor depresivo tratada con TEC, Mianserina y Amisulpiride ${ }^{(12)}$. McClenahan y Westphal (2006) reportaron el cuadro de un hombre de 52 años con Síndrome de Cotard asociado a catalepsia, negativismo, mutismo selectivo, mirada fija y alteraciones en la postura, que se benefició de TEC, Lorazepam y Venlafaxina ${ }^{(13)}$. Por otra parte, Yamada et al. (1999) presentaron una mujer de 46 años que cursó con delirios hipocondríacos y de negación de la existencia de ciertos órganos, asociado a negativismo y agitación, que respondió a TEC $^{(10)}$. De manera similar, Montgomery y Vasu (2007) publicaron el caso de una mujer de 48 años que respondió a TEC ${ }^{(14)}$; y Grover, Shah y Ghosh (2010) el de una mujer de 37 años que se benefició de TEC, Olanzapina y Venlafaxina (15). A diferencia de lo que muestra la literatura en estos casos, nuestra paciente evolucionó satisfactoriamente sin necesidad de TEC.

Siguiendo con los casos publicados en los últimos cinco años de pacientes con Depresión psicótica; Simpson, Kaul y Quinn (2013) describieron el de un hombre de 68 años con delirios hipocondríacos y nihilistas de existencia que posteriormente desarrolló excitación, mirada fija, ecopraxia, impulsividad y ambitendencia, que se benefició del uso de Olanzapina, Mirtazapina, Citalopram y Lorazepam $^{(8)}$. Basu, Singh, Gupta y Soni (2013) presentaron el caso de una mujer de 45 años con delirios nihilistas de existencia asociado a inmovilidad, estupor, mutismo, mirada fija y catalepsia que respondió a Sertralina, Olanzapina y Lorazepam $^{(16)}$. Una combinación distinta del cuadro fue descrita por Weiss, Santander y Barros (2013) reportando el caso de una mujer de 22 años con delirios nihilistas de existencia de partes de su cuerpo junto a inmovilidad, estupor, mutismo $\mathrm{y}$ negativismo, quien además presentó un Síndrome Neuroléptico Maligno tratado con TEC, Olanzapina y Lamotrigina $^{(2)}$. Finalmente, Huarcaya-Victoria y PodestáAmpuero (2018) publicaron el caso de un varón de 47 años con ideas delirantes nihilistas de existencia y de culpa, asociado a mutismo, flexibilidad cérea, ambitendencia y negativismo, que se benefició del uso de Venlafaxina, Aripiprazol y Diazepam ${ }^{(5)}$.

Además, se han encontrado casos con Síndrome de Cotard y Catatonía en el contexto de un Trastorno Bipolar con episodio depresivo reciente, como los señalados por Baeza, Salva y Bernardo (2000) en un hombre de 20 años con Síndrome de Cotard junto a inmovilidad, mutismo y rigidez muscular que requirió TEC e Imipramina ${ }^{(17)}$; y Grover, Aneja, Mahajan y Varma (2014) en una mujer de 62 años con delirios nihilistas de existencia y de inmortalidad, que posteriormente desarrolló mutismo, rigidez y negativismo, tratado con TEC, Olanzapina, Fluoxetina y estabilizadores del ánimo ${ }^{(18)}$. También se describió la comorbilidad en un hombre de 50 años con Esquizofrenia, delirios nihilistas de existencia, de culpa, condenación, inmortalidad, hipocondríacos y persecutorios, junto a inmovilidad, estupor, mutismo, mirada fija, rigidez y flexibilidad cérea, que respondió a Aripiprazol y Clonazepam a las 
dos semanas de tratamiento ${ }^{(19)}$.

Lo infrecuente de la asociación constituye una limitación a la hora de extraer conclusiones con respecto al tema, sin embargo, se aprecia alta concordancia en el tratamiento de ambos síndromes en que muchos de ellos recibieron benzodiazepinas y $\mathrm{TEC}^{(8)}$. Weiss et al. (2013) han señalado que desde una perspectiva neurobiológica, ambas condiciones clínicas implican vías en común (orbitofrontal y motora), lo que podría explicar ciertas similitudes en la sintomatología y respuesta a tratamiento ${ }^{(2)}$. Como hipótesis, Grover et al. (2014) plantean que el desarrollo de una Catatonía en el contexto de un paciente con Síndrome de Cotard, podría deberse a un proceso de progresión de éste último y de la Depresión psicótica del caso que presentaron ${ }^{(18)}$. Los elementos planteados por Weiss et al. (2013) y Grover et al. (2014) resultan de gran interés a la hora de establecer hipótesis que permitan esclarecer el modo en que se da la aparición conjunta de los dos síndromes, así, ambas posturas pudieran ser complementarias.

Se requieren más estudios que permitan aclarar esta relación, lo que implica un conocimiento más acabado de la patogenia, clínica y terapéutica de ambos síndromes. En este sentido, es fundamental contar con sólidos conocimientos en psicopatología y una búsqueda activa de ambas entidades clínicas, para así llegar a establecer criterios diagnósticos que nos encaminen a investigar con mayor precisión sobre su etipatogenia.

\section{Resumen}

El Sindrome de Cotard es una condición neuropsiquiátrica poco frecuente, descrito inicialmente por Jules Cotard como un delirio hipocondríaco y luego como Delirio de negación, en que el paciente niega la existencia de partes de su cuerpo, la propia existencia y/o del mundo entero. La aparición de un Síndrome Catatónico junto al Síndrome de Cotard es aún más infrecuente. Se presenta el caso de una paciente de 72 años con una Depresión psicótica, que desarrolla un Síndrome de Cotard y posteriormente Catatonía. Logra buena respuesta tras la adición de Lorazepam y Venlafaxina al esquema farmacológico en curso, por lo que se desestima el uso de Terapia Electroconvulsiva. Se constata remisión total de sintomas y posterior recuperación funcional ad integrum, siendo evaluada a través de entrevista clinica, Hamilton Depression Rating Scale, Bush-Francis Catatonia Rating Scale e Índice de Barthel. Además, se revisan otros reportes de caso sobre esta comorbilidad, y a diferencia de la mayoría de éstos, se destaca la favorable evolución de la paciente sin necesidad de Terapia Electroconvulsiva. Aún no se ha dilucidado la relación entre ambos síndromes, aunque algunos autores han planteado la hipótesis de vías neurobiológicas compartidas yotros han postulado la aparición de síntomas catatónicos como la progresión del Sindrome de Cotard. Para aclarar estas interrogantes, son necesarios más estudios al respecto que permitan conocer la etiopatogenia de esta inusual combinación.

Palabras claves: Sindrome Cotard, Catatonía, Depresión. 


\section{Referencias Bibliográficas}

1. Álvarez J, Colina F, Esteban R. Presentación de Delirios Melancólicos: Negación y Enormidad. En: Cotard J, Séglas J. Delirios Melancólicos: Negación y Enormidad (selección). Madrid: Alienistas del Pisuerga, 2008; p. XI-XXXIII.

2. Weiss C, Santander J, Torres R. Catatonia, neuroleptic malignant syndrome, and Cotard syndrome in a 22-yearold woman: A Case Report. Case Rep Psychiatry 2013; 2013: 452646. http:// dx.doi.org/10.1155/2013/452646.

3. Dieguez S. Cotard Syndrome. Front Neurosci 2018; 42: 23-34. doi: $10.1159 / 000475679$.

4. Machado L, Filho LE, Machado L. When the Patient Believes That the Organs are Destroyed: Manifestation of Cotard's Syndrome. Case Rep Med 2016; 2016: 5101357. http://dx.doi. org/10.1155/2016/5101357.

5. Huarcaya-Victoria J, Podestá-Ampuero A. Síndrome de Cotard, catatonía y depresión: reporte de un caso. Rev

6. Neuropsiquiatr 2018; 81 (2): 135-140. Rasmussen S, Mazurek M, Rosebush P. Catatonia: Our current understanding of its diagnosis, treatment and pathophysiology. World J Psychiatr 2016; 6 (4): 391-398.

7. Walher S, Strik W. Catatonia. CNS Spectr 2016; 21 (4): 341-348.

8. Simpson P, Kaul E, Quinn D. Cotard's Syndrome with Catatonia: A case Presentation and Discussion. Psychosomatics 2013; 54 (2): 196-199.

9. Berrios G, Luque R. Cotard's syndrome: analysis of 100 cases. Acta Psychiatr Scand 1995; 91 (3): 185-188.

10. Yamada K, Katsuragi S, Fujii I. A case study of Cotard's syndrome: stages and diagnosis. Acta Psychiatr Scand 1999; 100 (5): 396-399.
11. Ramírez-Bermudez J, Agular-Venegas L, Crail-Melendez D, Espinola-Nadurille M, Nente F, Mendez F. Cotard's síndrome in neurological and psychiatric patients. J Neuropsychiatry Clin Neurosci 2010. 22 (4): 409-416.

12. Cohen D, Cottias C, Basquin M. Cotard's syndrome in a 15-year-old girl. Acta Psychiatr Scand 1997; 95 (2): 164-165.

13. McClenahan E, Westphal J. Depressed, delusional, and "dead". Current Psychiatry 2006; 5(7): 105-113.

14. Montgomery J, Vasu D. The use of electroconvulsive therapy in atypical psychotic presentations: a case review. Psychiatry (Edgmont) 2007; 4 (10): 30-39.

15. Grover S, Shah R, Ghosh A. Electroconvulsive therapy for lycanthropy and Cotard's syndrome: a case report. J ECT 2010; 26 (4): 280-281.

16. Basu A, Singh P, Gupta R, Soni S. Cotard Syndrome with Catatonia: Unique Combination. Indian J Psychol Med 2013; 35 (3): 314-316.

17. Baeza I, Salva J, Bernardo M. Cotard's syndrome in a young male bipolar patient. J Neuropsychiatry Clin Neurosci 2000; 12 (1): 119-120.

18. Grover S, Aneja J, Mahajan S, Varma S. Cotard's syndrome: Two case reports and a brief review of literature. J Neurosci Rural Pract 2014; 5 (1): 5962.

19. Huarcaya-Victoria J, Ledesma-Gastañadui M, Huete-Cordova M. Cotard's Syndrome in a patient with schizophrenia: case report and review of the literature. Case Rep Psychiatry. 2016; 2016: 6968409. 
Sergio Vergara R, Et. Al.

Correspondencia:

Sergio Andrés Vergara Ramírez.

Edificio Plaza Poniente 1258, oficina 209,

Talca.

vergarasergio1@gmail.com,

cel: 9-85493299, cel: 9-42649222. 